\title{
Level of Intended Sedation
}

\author{
G.A. Paspatis $^{\mathrm{a}} \quad$ G. Tribonias ${ }^{\mathrm{a}} \quad$ K. Paraskeva ${ }^{\mathrm{b}}$ \\ ${ }^{a}$ Department of Gastroenterology, Benizelion General Hospital, Heraklion, and \\ ${ }^{b}$ Department of Gastroenterology, Konstantopoulio-Agia Olga General Hospital, Athens, Greece
}

\section{Key Words \\ Gastrointestinal endoscopy · Sedation · Analgesia}

\begin{abstract}
Sedation and analgesia is the standard of care during diagnostic and therapeutic endoscopic gastrointestinal procedures in most areas of the world. Unsedated endoscopy is feasible in selected patients, but requires commitment on the part of both the patient and the provider. The American Society of Anesthesiologists has defined four stages of sedation, ranging from minimal to moderate, deep and general anesthesia. The level of sedation as well as the choice of sedative is based on the type of procedure, patient characteristics and the existence of the required structural conditions. Individuals administering sedation/analgesia should be trained to rescue a patient who has reached a level of sedation deeper than that intended.
\end{abstract}

Copyright $\odot 2010$ S. Karger AG, Basel

\section{Introduction}

Gastrointestinal endoscopy remains an unpleasant procedure without sedation. Therefore, sedation should be offered to every patient before the procedure. The purpose of sedation and analgesia is to relieve patient anxiety and discomfort, improve the outcome of the examination, and diminish the patient's memory of the event.
Sedation and analgesia is the standard of care during diagnostic and therapeutic endoscopic gastrointestinal procedures in most areas of the world. Unsedated endoscopy is feasible in selected patients, but requires commitment from both the patient and the provider $[1,2]$. With purely diagnostic gastroscopy, the use of sedatives involves a slightly higher rate of complications, but patient acceptance is significantly higher than without [3]. Every patient has the right to an endoscopic examination that is as painless and stress-free as possible. Indeed, there are great variations worldwide as far as the sedation for the gastrointestinal endoscopy is concerned. However, recent surveys show an increase in sedation frequency for endoscopic procedures [4-10].

The use of sedation/analgesia raised the likelihood of completing the colonoscopy. Radaelli et al. [9] compared colonoscopies done with and without sedation/analgesia. It appeared that intravenous benzodiazepine increased the probability of reaching the cecum by $46 \%$, and the combination of benzodiazepine + narcotic i.v. or propofol increased it even more. Moreover, the use of sedation/analgesia was significantly associated with the likelihood of detecting at least one polyp by colonoscopy. This finding shows that sedation/analgesia not only improves patient acceptance of the procedure, but also the overall quality of the examination, assessed on the basis of cecum intubation and polyp detection.

\section{KARGER}

Fax +4161306 1234

E-Mail karger@karger.ch

www.karger.com
(C) 2010 S. Karger AG, Basel

$0012-2823 / 10 / 0822-0084 \$ 26.00 / 0$

Accessible online at:

www.karger.com/dig
G.A. Paspatis, MD

Department of Gastroenterology

Benizelion General Hospital, L. Knossou

GR-71409 Heraklion (Greece)

Tel./Fax +30 2810368 017, E-Mail paspati@admin.teiher.gr 


\section{Levels of Sedation}

The American Society of Anesthesiologists (ASA) has described four stages of sedation, ranging from minimal to moderate, deep and general anesthesia [11]. Minimal sedation (anxiolysis) signifies a drug-induced state in which patients respond normally to verbal commands. Although cognitive function and coordination may be impaired, ventilatory and cardiovascular functions are unaffected. Moderate sedation/analgesia (conscious sedation) signifies a drug-induced depression of consciousness during which patients respond purposefully to verbal commands, either alone or accompanied by light tactile stimulation. No interventions are required to maintain a patent airway and spontaneous ventilation is adequate. Cardiovascular function is usually maintained. Deep sedation/analgesia signifies a drug-induced depression of consciousness during which patients cannot be easily aroused, but respond purposefully following repeated or painful stimulation. The ability to independently maintain ventilatory function may be impaired. Patients may require assistance in maintaining a patent airway, and spontaneous ventilation may be inadequate. Cardiovascular function is usually maintained. General anesthesia signifies a drug-induced loss of consciousness during which patients are not arousable, even by painful stimulation. The ability to independently maintain ventilatory function is often impaired. Patients often require assistance in maintaining a patent airway, and positive pressure ventilation may be required because of depressed spontaneous ventilation or drug-induced depression of neuromuscular function. Cardiovascular function may be impaired.

\section{Procedure-/Patient-Oriented Level of Sedation}

The level of the sedation as well as the choice of sedative is based on the type of procedure, patient characteristics and existence of the required structural conditions $[10,12,13]$. Diagnostic and therapeutic gastroscopies and colonoscopies are successfully performed with moderate sedation. More complex procedures such as ERCP and EUS are successfully performed with deep sedation. Even for a purely diagnostic endoscopy, sedation can be advantageous, not only for the patient, but also in terms of achievement of higher-quality procedures. The following list summarizes the endoscopic procedures which most often require the use of sedation or deep sedation [3]: interventional endoscopy in the upper gastrointestinal tract (hemostasis; treatment of varices with ligation, sclerotherapy, or tissue-adhesive therapy; dilation procedures; implantation of prostheses; endoscopic mucosal resection; and disobliteration procedures); diagnostic and interventional endosonography; percutaneous endoscopic gastrostomy; colonoscopy with difficult and/or multiple polypectomy or with endoscopic mucosal resection; and ERCP with interventions involving the bile ducts (endoscopic papillotomy, mechanical lithotripsy, stone removal, implantation of prostheses) and the pancreatic ducts (selective sphincterotomy, stone removal, dilation, implantation of prostheses).

Deep sedation or general anesthesia might be considered in selected patients, including those who have had long-term use of narcotics, benzodiazepines, alcohol or neuropsychiatric medications. Before the procedure, societies recommend a risk assessment of possible cardiovascular and respiratory disease, whereby the ASA should be considered [ASA classification of anesthetic risk according to general health status ( $\mathrm{I}=$ healthy patient, $\mathrm{II}=$ mild illness without physical limitations, III = serious illness with physical limitations, IV = serious illness with threat to life, $\mathrm{V}=$ death expected within $24 \mathrm{~h}$ )] [11, 13].

\section{Unintended Level of Deep Sedation}

Deep sedation may occur during endoscopy using the combination of benzodiazepines and opioids intended to reach moderate sedation [14]. If propofol is administered as the sole agent, deep sedation may be required to keep the patient comfortable [13]. A patient targeted for one level of sedation may become more deeply sedated than planned. Specifically, deep sedation occurs frequently during elective endoscopy with midazolam and narcotics used with the intent of moderate sedation. Patel et al. [14] demonstrated that deep sedation episodes of all sedationlevel observations by procedure was $26 \%$ for EGD, $11 \%$ for colonoscopy, 35\% for ERCP and 29\% for EUS. From the same study, deep sedation occurred at least once in $60 \%$ of EGD, $45 \%$ of colonoscopy, $85 \%$ of ERCP and $80 \%$ of EUS. It is noteworthy that ERCP and EUS are risk factors for the occurrence of deep sedation, independent of sedation dose or length of procedure [14].

Individuals administering sedation/analgesia should be trained to rescue a patient who has reached a level of sedation deeper than that intended [13]. Because sedation is a continuum, it is not always possible to predict how an individual patient will respond to intravenous sedatives. Hence, practitioners should be able to rescue patients 
whose level of sedation becomes deeper than initially intended. Individuals administering moderate sedation/ analgesia (conscious sedation) should be able to rescue patients who enter a state of deep sedation/analgesia, while those administering deep sedation/analgesia should be able to rescue patients who enter a state of general anesthesia [11]. The patient's level of consciousness should be followed throughout the endoscopy with scales such as MOAAS or Ramsay.

In all cases when a patient has an increased risk, or when a long, complex intervention is expected, a second person qualified in resuscitation and intensive care with the sole task of administering the sedation and monitor- ing the patient should be present $[3,10,12]$. According to published data, this person can be an anesthesiologist, a specially trained physician or a specially trained member of the nursing staff [3]. Physicians who are not anesthesiologists are not allowed to perform sedation and/or analgesia procedures that would reach a planned degree of sedation that affects or eliminates life-preserving reflexes. If, in the occasional case, it happens that a degree of sedation is reached that affects or eliminates life-preserving reflexes (general anesthesia), and if the intervention is to be continued, an anesthesiologist should be called in [10].

\section{References}

1 Eckardt VF, Kanzler G, Schmitt T, Eckardt AJ, Bernhard G: Complications and adverse effects of colonoscopy with selective sedation. Gastrointest Endosc 1999;49:560-565.

$>2$ Rex DK, Imperiale TF, Portish V: Patients willing to try colonoscopy without sedation: associated clinical factors and results of a randomized controlled trial. Gastrointest Endosc 1999;49:554-559.

$>3$ Schreiber F: Austrian Society of Gastroenterology and Hepatology (OGGH) - guidelines on sedation and monitoring during gastrointestinal endoscopy. Endoscopy 2007;39:259-262.

$\checkmark 4$ Baudet JS, Borque P, Borja E, et al: Use of sedation in gastrointestinal endoscopy: a nationwide survey in Spain. Eur J Gastroenterol Hepatol 2009;21:882-888.
5 Cohen LB, Wecsler JS, Gaetano JN, et al: Endoscopic sedation in the United States: results from a nationwide survey. Am J Gastroenterol 2006;101:967-974.

6 Heuss LT, Froehlich F, Beglinger C: Changing patterns of sedation and monitoring practice during endoscopy: results of a nationwide survey in Switzerland. Endoscopy 2005;37:161-166

$>7$ Ladas SD, Aabakken L, Rey JF, et al: Use of sedation for routine diagnostic upper gastrointestinal endoscopy: a European Society of Gastrointestinal Endoscopy Survey of National Endoscopy Society Members. Digestion 2006;74:69-77.

$>8$ Paspatis GA, Manolaraki MM, Tribonias G, et al: Endoscopic sedation in Greece: results from a nationwide survey for the Hellenic Foundation of Gastroenterology and Nutrition. Dig Liver Dis 2009;41:807-811.

$>9$ Radaelli F, Meucci G, Sgroi G, Minoli G: Technical performance of colonoscopy: the key role of sedation/analgesia and other quality indicators. Am J Gastroenterol 2008; 103:1122-1130.
10 Riphaus A, Wehrmann T, Weber B, et al: S3 Guideline: sedation for gastrointestinal endoscopy 2008. Endoscopy 2009;41:787-815.

11 American Society of Anesthesiologists Task Force on Sedation and Analgesia by NonAnesthesiologists: Practice guidelines for sedation and analgesia by non-anesthesiologists. Anesthesiology 2002;96:1004-1017.

12 Lichtenstein DR, Jagannath S, Baron TH, et al: Sedation and anesthesia in GI endoscopy. Gastrointest Endosc 2008;68:815-826.

13 Cohen LB, Delegge MH, Aisenberg J, et al: AGA Institute review of endoscopic sedation. Gastroenterology 2007;133:675-701.

14 Patel S, Vargo JJ, Khandwala F, et al: Deep sedation occurs frequently during elective endoscopy with meperidine and midazolam. Am J Gastroenterol 2005; 100:2689-2695. 